\title{
Antihormonal treatment associated musculoskeletal pain in women with breast cancer in the adjuvant setting
}

This article was published in the following Dove Press journal:

OncoTargets and Therapy

9 August 2016

Number of times this article has been viewed

\author{
Selcuk Seber ${ }^{1}$ \\ Dilek Solmaz ${ }^{2}$ \\ Tarkan Yetisyigit ${ }^{\prime}$ \\ 'Medical Oncology Department, \\ ${ }^{2}$ Rheumatology Department, \\ Namik Kemal University Hospital, \\ Tekirdag, Turkey
}

Correspondence: Selcuk Seber Medical Oncology Department, Namik Kemal University Hospital, University Street, Suleymanpasa,

Tekirdag 59000, Turkey

Tel +90282 250 54II

Fax +90282 2509928

Email selcukseber@gmail.com
Purpose: Antihormonal treatment is an effective therapy in the adjuvant setting. However, musculoskeletal pain is a common adverse effect encountered in patients receiving this treatment. We aimed to evaluate the risk factors for the development of antihormonal treatmentassociated musculoskeletal pain (AHAMP) and its impact on the health-related quality of life (HRQOL).

Patients and methods: A cross-sectional survey of 78 consecutive breast cancer patients receiving adjuvant antihormonal treatment for early-stage breast cancer in an academic medical oncology clinic was conducted. AHAMP was assessed by Health Assessment Questionnaire (HAQ) and $10 \mathrm{~cm}$ visual analog scale (VAS). HRQOL was assessed by self-administered short form 36 and Functional Assessment of Cancer Therapy-Breast subscale surveys.

Results: AHAMP was found to be present in 37 (47.7\%) patients. In multivariate regression analysis, having a normal body mass index $\left(<30 \mathrm{~kg} / \mathrm{m}^{2}\right)$, cigarette smoking, and low serum vitamin D level $(20 \mathrm{ng} / \mathrm{mL})$ were found to be independent risk factors. In HRQOL assessment, physical and mental scores were found to be significantly lower in patients with joint arthralgia.

Conclusion: AHAMP has an adverse effect on the quality of life of breast cancer patients receiving adjuvant antihormonal treatment, and assessment of predictive factors is important for identification of patient groups at risk of developing this condition.

Keywords: antineoplastic hormonal agents, musculoskeletal pain, breast cancer

\section{Introduction}

Antihormonal treatment for breast cancer is currently the cornerstone of modern adjuvant therapy in hormone receptor positive cancer patients. The success of adjuvant treatments leads to an improvement in long-term survival rates, and quality of life issues in breast cancer survivors have become an important aspect in the management of these patients. Most of the side effects related to the antihormonal treatment are generally thought to be derived from estrogen deprivation. Joint pain and arthralgia are among the most commonly encountered side effects seen in patients receiving tamoxifen or aromatase inhibitors (AIs) as hormonal adjuvant treatment.

The incidence of joint-related symptoms (joint pain, stiffness, arthralgia) associated with antihormonal treatment has been analyzed in various large randomized studies. In an analysis of BIG 1-98 trial, which incorporated more than 8,000 postmenopausal breast cancer patients, nearly $20 \%$ of women randomized to letrozole therapy experienced arthralgia while the incidence was $13.4 \%$ in women who received tamoxifen. ${ }^{1}$ The incidence of bone and joint pain in patients receiving additional 
ovarian function suppression therapy is reported to be $20 \%$ in large randomized trials. ${ }^{2}$ However, in several retrospective or cross-sectional trials that specifically surveyed treatmentrelated musculoskeletal adverse effects, the incidence of joint-related adverse effects was reported to be between 30\% and $66 \%$. Moderate to high-grade joint-related symptoms are also associated with poor adherence to therapy or treatment discontinuation, besides interfering with the daily activities of the survivors. ${ }^{3-5}$

The aim of this study was to evaluate the precipitating factors that are behind the musculoskeletal symptoms associated with antihormonal therapy and also assess the impact of these drug-related adverse effects on patients' quality of life.

\section{Methods}

\section{Study population}

Breast cancer patients who were being treated with adjuvant antihormonal therapy in a university hospital medical oncology clinic were included in the study group. All patients receiving steroidal AIs (anastrozole or letrozole) or combination antihormonal treatment with tamoxifen and monthly injections of $3.6 \mathrm{mg}$ goserelin were eligible. Among the patients attending the oncology clinic, subjects who provided informed consent were recruited consecutively. All the participants were receiving an antihormonal therapy for at least 30 days at the time of interview. Early-stage breast cancer (stage I-III) patients who had undergone planned surgery for breast cancer and completed adjuvant chemotherapy and radiotherapy treatments were included in the study group. The following patients were excluded from the study group: those with advanced stage breast cancer, those with a known second primary cancer, patients who had been diagnosed with a rheumatological disease and were actively treated for it, and patients with a known bone metabolic disease.

\section{Data collection}

A cross-sectional survey was designed to gather data from the participating patients. After informed consent was taken, patients were asked to complete a one-time self-administered survey about joint symptoms, treatments received, and demographic variables. Then they were thoroughly examined by an experienced rheumatologist for the presence of musculoskeletal symptoms. Musculoskeletal symptoms that initiated after the antihormonal treatment or exacerbated after initiation of the endocrine therapy were recorded. Antihormonal treatment associated musculoskeletal pain (AHAMP) was defined as follows: symmetrical arthralgia affecting wrists, hands, and knees, arthritis, tenosynovitis, myalgia, and trigger finger, which have developed since starting antihormonal treatment. ${ }^{6}$

\section{Measures}

The severity of the pain was assessed by both visual analog scale (VAS) $(0-10 \mathrm{~cm})$ and Heath Assessment Questionnaire (0-3). Health-related quality of life (HRQOL) was assessed by both Medical Outcomes Study short form 36 Version 2 (SF-36) and Functional Assessment of Cancer Therapy-Breast (FACT-B) subscale. SF-36 is widely used as a measure of life quality in a variety of patient populations and is also validated for Turkish cancer patients. ${ }^{7}$ Both SF-36 and FACT-B measure physical and mental well-being. Higher scores in both questionnaires indicate better HRQOL. In addition, FACT-B has a separate breast cancer subscale (BCS) that is designed specifically for assessment of HRQOL in patients with breast cancer. FACT-General score constitutes sum of the scores of physical well-being, functional well-being, social/family well-being, and emotional well-being components. FACT-B total score is obtained by addition of FACT-General and BCS scores. ${ }^{8}$ License for a validated Turkish translation version of the questionnaire was obtained from www.FACIT.org.

At the time of interview, demographic information regarding patient's social status, age, education level, current medications, and cigarette smoking was obtained from a self-report questionnaire. At the same time, blood samples were taken for assessment of serum 25-hydroxy vitamin D (25(OH)D), C-reactive protein, and erythrocyte sedimentation rate levels. All biomarkers were evaluated using standard laboratory techniques. Written consent was obtained from all of the patients for inclusion of their data in this study prior to administering the tool. The study was approved by the Namik Kemal University Hospital ethics committee.

\section{Statistical analysis}

Statistical analyses were done by utilizing Statistical Package for the Social Sciences 16.0 (SPSS Inc., Chicago, IL, USA). Continuous variables are expressed as mean and standard deviation or median minimum and maximum values, while categorical variables are expressed as percentages. Continuous variables between groups were examined with Mann-Whitney $U$ statistic and categorical variables with chi-square test. Related factors for joint symptoms and pain were tested using logistic regression; results were summarized using odds ratios and $95 \%$ confidence intervals. Each characteristic was first tested in a univariate analysis and then all variables that were statistically significant at a level of $P<0.10$ were included in the multivariate analysis. 
Enter method was used in multivariate analysis. $P$-value $<0.05$ was accepted as statistically significant.

\section{Results}

Among 95 eligible women attending the medical oncology clinic with early-stage breast cancer, 78 patients who accepted to participate and gave informed consent were included in the study group. Thirty-seven (47.4\%) patients were found to have musculoskeletal symptoms associated with antihormonal treatment. Median duration of antihormonal treatment at the time of interview was 16 months (4-69 months). Mean time period for the initiation of musculoskeletal pain was 2 months (1-10 months).

Demographic variables of the patient group are summarized in Table 1. Fifty-four postmenopausal women were receiving nonsteroidal AIs, while 24 premenopausal women received tamoxifen in combination with a luteinizing hormone releasing hormone (LHRH) agonist (monthly injections of $3.6 \mathrm{mg}$ goserelin acetate). The mean age of the patients with AHAMP was significantly lower compared to that of patients without symptoms (50.6 vs 55.4, $P=0.028$ ). Also, mean body mass index (BMI) was significantly lower in patients with AHAMP (28.7 vs 30.6, $P=0.036$ ). Mean time from the onset of menopause was longer in patients with AHAMP; however, this difference did not reach statistical

Table I Demographic variables of patients with and without AHAMP

\begin{tabular}{|c|c|c|}
\hline Demographic variable & $\begin{array}{l}\text { AHAMP } \\
(n=37)\end{array}$ & $\begin{array}{l}\text { No AHAMP } \\
(n=4 I)\end{array}$ \\
\hline Age, mean $\pm S D$ & $50.6 \pm 10.8 *$ & $55.4 \pm 9.7$ \\
\hline Cigarette smoking ever, n; \% & $15 ; 40.5^{*}$ & $8 ; 19.5$ \\
\hline $\begin{array}{l}\text { No of children, median } \\
\text { (minimum-maximum) }\end{array}$ & $2(0-5)$ & $2(0-4)$ \\
\hline Education, mean \pm SD (years) & $6 \pm 2.8$ & $6 \pm 2.6$ \\
\hline $\begin{array}{l}\text { Time from onset of } \\
\text { menopause, mean } \pm \text { SD (years) }\end{array}$ & $12 \pm 10.3$ & $9 \pm 8.9$ \\
\hline $\mathrm{BMI}$, mean $\pm \mathrm{SD}$ & $28.7 \pm 3.6 *$ & $30.6 \pm 4.2$ \\
\hline I8-24.9 (normal), n; \% & $5 ; 13.5$ & $3 ; 7.3$ \\
\hline 25.0-29.9 (overweight), n; \% & $19 ; 51.3$ & $15 ; 36.5$ \\
\hline 30 (obesity), n; \% & II; 29.7* & $19 ; 46.3$ \\
\hline \multicolumn{3}{|l|}{ Stage } \\
\hline I, n; \% & $3 ; 8.1$ & $6 ; 14.6$ \\
\hline II, n; \% & $18 ; 48.6$ & $24 ; 58.5$ \\
\hline III, n; \% & $16 ; 43.2$ & II; 26.9 \\
\hline \multicolumn{3}{|l|}{ Antihormonal treatment } \\
\hline Tamoxifen + goserelin, n; \% & $8 ; 21.6$ & $16 ; 39.0$ \\
\hline Anastrozole, n; \% & $14 ; 37.8$ & $15 ; 36.6$ \\
\hline Letrozole, n; \% & $15 ; 40.5$ & $10 ; 24$ \\
\hline
\end{tabular}

Note: *AHAMP versus no AHAMP, $P<0.05$.

Abbreviations: AHAMP, antihormonal treatment associated musculoskeletal pain; BMI, body mass index; SD, standard deviation. significance ( 12 vs 9 years, $P=0.787$ ). Musculoskeletal pain was significantly more common in patients who smoked (40.5\% vs $19.5 \%, P=0.002)$. There was a trend toward higher incidence of presence of AHAMP in patients receiving letrozole, when compared to patients receiving tamoxifen plus LHRH agonist therapy; however, this difference did not reach statistical significance $(P=0.062)$.

All patients had early-stage disease (I-III) and were operated for their primary lesion. All of the participating patients received prior chemotherapy with sequential anthracycline and taxane regimens.

Serum 25(OH)D levels were available from $66(84 \%)$ patients. Mean serum 25(OH)D level was $21.2 \mathrm{ng} / \mathrm{mL} \pm 7.1$ and $46 \%$ of the participants had $25(\mathrm{OH}) \mathrm{D}$ deficiency $(<20 \mathrm{ng} / \mathrm{mL})$. Serum 25(OH)D levels were found to be significantly lower (18.2 vs $24.4 \mathrm{ng} / \mathrm{mL}, P=0.013)$ in patients with musculoskeletal pain, while other laboratory parameters were similar between the two groups (Table 2).

In both univariate and multivariate regression analyses, serum 25(OH)D levels, cigarette smoking, and BMI were found to be the independent risk factors for developing musculoskeletal pain under antihormonal treatment for breast cancer (Table 3).

A high correlation between VAS and Heath Assessment Questionnaire scores was present $(P<0.001, r=0.643)$. Age and BMI were found to be negatively correlated with pain intensity (VAS pain vs age: $P=0.002, r=-0.493$; VAS pain vs BMI: $P=0.003, r=-0.490)$, while low serum 25(OH)D levels and smoking status were not found to be associated with pain intensity. When a linear regression analysis was made, only

Table 2 Laboratory variables of patients with and without AHAMP

\begin{tabular}{lll}
\hline Laboratory data & $\begin{array}{l}\text { AHAMP } \\
(\mathbf{n}=\mathbf{3 7})\end{array}$ & $\begin{array}{l}\text { No AHAMP } \\
(\mathbf{n}=\mathbf{4 I})\end{array}$ \\
\hline CRP, mean \pm SD & $4.5 \pm 4.0$ & $4.2 \pm 2.8$ \\
ESR, mean \pm SD & $25 \pm 28.0$ & $18.2 \pm 10.5$ \\
CK, mean \pm SD & $92 \pm 42.6$ & $91 \pm 62.6$ \\
RF, mean \pm SD & $10 \pm 8.8$ & $8 \pm 3.4$ \\
Serum 25(OH)D, mean \pm SD & $18.2 \pm 8.9 *$ & $24.4 \pm 9.9$ \\
$\leq 10$ ng/mL (deficient), n; \% & $8 ; 21.6^{*}$ & 0 \\
I0-20 ng/mL (insufficient), n; \% & $11 ; 29.7^{*}$ & $17 ; 41.4$ \\
$>20$ ng/mL (optimal), n; \% & $15 ; 40.5$ & $15 ; 36.5$ \\
ANA positivity, n; \% & 0 & NA \\
Anti-CCP positivity, n; \% & 0 & NA \\
\hline
\end{tabular}

Note: *AHAMP versus no AHAMP, $P<0.05$.

Abbreviations: $25(\mathrm{OH}) \mathrm{D}$, 25-hydroxy vitamin D; AHAMP, antihormonal treatment associated musculoskeletal pain; ANA, antinuclear antibody; anti-CCP, anticyclic citrullinated peptide; CK, creatinine kinase; CRP, C-reactive protein; ESR, erythrocyte sedimentation rate; NA, not applicable; RF, rheumatoid factor; SD, standard deviation. 
Table 3 Multivariate logistic regression analysis for determination of AHAMP risk factors

\begin{tabular}{llll}
\hline Variables & P-value & OR & 95\% Cl \\
\hline Age & 0.089 & 1.055 & $0.992-1.121$ \\
D vitamin level $(\geq 20 \mathrm{ng} / \mathrm{mL}$ & 0.003 & 0.899 & $0.839-0.964$ \\
vs $<20 \mathrm{ng} / \mathrm{mL}$ ) & & & \\
$\mathrm{BMI}(\geq 30 \mathrm{vs}<30)$ & 0.002 & 0.915 & $0.898-0.978$ \\
$\begin{array}{l}\text { Cigarette smoking } \\
\text { (nonsmoker vs smoker) }\end{array}$ & 0.010 & 0.125 & $0.026-0.608$ \\
\hline
\end{tabular}

Abbreviations: AHAMP, antihormonal treatment associated musculoskeletal pain; $\mathrm{BMI}$, body mass index; $\mathrm{Cl}$, confidence interval; OR, odds ratio.

age factor was found to be independently correlated with pain intensity (regression coefficient: -0.346 [95\% confidence interval: -0.103 to 0.000 ]; $P<0.05$ ) (Figure 1 ).

Patients with AHAMP had significantly lower HRQOL scores in all aspects of the FACT-B questionnaire (physical, emotional, social, and functional well-being, and BCS) compared to patients without symptoms, while only physical component score of the SF-36 was significantly lower in patients with pain (Table 4).

\section{Discussion}

We observed that nearly half of the women who were diagnosed with breast cancer experienced musculoskeletal pain related to antihormonal treatment given in the adjuvant setting. We documented that these symptoms significantly interfered with the quality of life of the patients affected by the antihormonal treatment.

In BIG 1-98 randomized clinical trial, which directly compared the clinical effectiveness of tamoxifen and

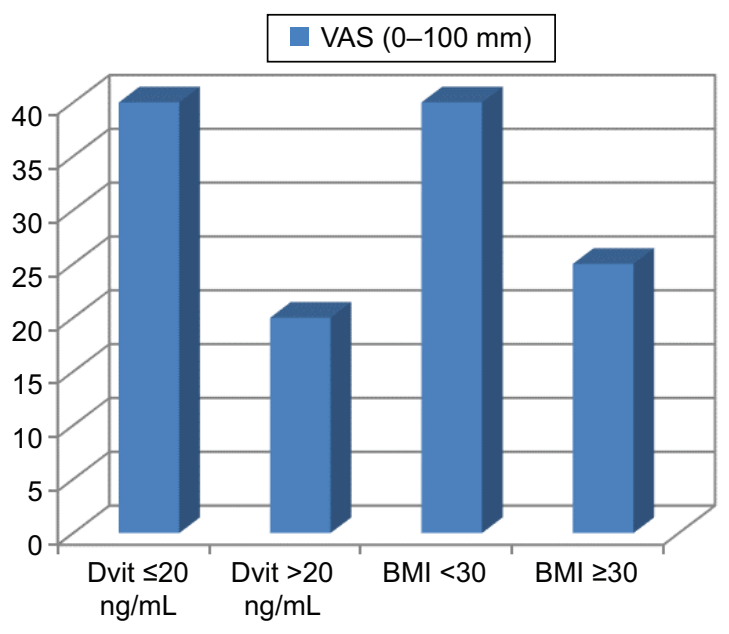

Figure I The impact of serum 25(OH)D level and BMI on severity of joint pain represented by VAS scores.

Abbreviations: 25(OH)D, 25-hydroxy vitamin D; BMI, body mass index; VAS, visual analog scale; Dvit, 25-hydroxy vitamin D.
Table 4 Pain intensity (VAS pain, HAQ) and health-related quality of life (FACT-B and SF-36) scores in patients with and without AHAMP

\begin{tabular}{lll}
\hline Observed scores & $\begin{array}{l}\text { AHAMP } \\
(\mathbf{n}=\mathbf{3 7})\end{array}$ & $\begin{array}{l}\text { No AHAMP } \\
(\mathbf{n}=\mathbf{4 1})\end{array}$ \\
\hline VAS pain & $5.3 \pm 1.7$ & $1.0 \pm 1.7$ \\
HAQ & $1.0 \pm 0.7$ & $0.2 \pm 0.4$ \\
SF-36 & & \\
$\quad$ Physical function & $37.3 \pm 11.9 *$ & $44.3 \pm 10.7$ \\
Role physical & $40.0 \pm 11.7$ & $42.4 \pm 11.8$ \\
Bodily pain & $39.3 \pm 9.9 * *$ & $49.8 \pm 10.4$ \\
General health & $42.9 \pm 10.5 * *$ & $49.5 \pm 9.2$ \\
Vitality & $46.4 \pm 12.9$ & $52.1 \pm 11.3$ \\
Social function & $40.4 \pm 11.7 * *$ & $49.6 \pm 7.3$ \\
Role emotion & $37.0 \pm 9.7$ & $35.5 \pm 11.8$ \\
Mental health & $43.9 \pm 11.8$ & $49.4 \pm 10.5$ \\
Physical component score & $35.8 \pm 10.0 * *$ & $47.4 \pm 9.1$ \\
Mental component score & $43.0 \pm 10.1$ & $46.0 \pm 9.3$ \\
FACT-B subscale & & \\
Physical well-being & $15.9 \pm 5.2 * *$ & $23.1 \pm 5.5$ \\
Social well-being & $20.5 \pm 4.9 * *$ & $23.1 \pm 3.8$ \\
Emotional well-being & $16.4 \pm 5.2 *$ & $20.0 \pm 4.2$ \\
Functional well-being & $18.3 \pm 4.5 * *$ & $21.1 \pm 5.2$ \\
Breast cancer subscale & $20.8 \pm 6.6 * *$ & $27.9 \pm 7.1$ \\
FACT-B total score & $18.3 \pm 4.0 * *$ & $23.1 \pm 4.3$ \\
FACT-G & $17.8 \pm 3.8 * *$ & $21.9 \pm 3.9$ \\
\hline Notes & &
\end{tabular}

Notes: *AHAMP versus no AHAMP, $P<0.05$. **AHAMP versus no AHAMP, $P<0.001$. Data presented as mean \pm SD.

Abbreviations: AHAMP, antihormonal treatment associated musculoskeletal pain; FACT-B, Functional Assessment of Cancer Therapy-Breast; FACT-G, Functional Assessment of Cancer Therapy-General; HAQ, Health Assessment Questionnaire; SD, standard deviation; SF-36, short form 36; VAS, visual analog scale.

letrozole in postmenopausal women, arthralgia and myalgia were reported to be more commonly observed in the letrozole group. We also observed that tamoxifen was less commonly associated with AHAMP; however, this did not reach statistical significance $(P=0.062)$. The rate of AHAMP $(21 \%$ and $40 \%$ for tamoxifen and letrozole, respectively) was higher when compared to the results reported in BIG 1-98 trial ( $13.5 \%$ and $20 \%$, respectively). However, it is stated by the authors that the rates of arthralgia and myalgia could have been underestimated due to the data gathering method used in this trial. ${ }^{1}$ Another important factor for this observation could be that the patients in our study received ovarian suppression with an LHRH agonist along with tamoxifen treatment. Loss of ovarian function due to LHRH suppression could have led to a higher incidence of joint-related symptoms in the tamoxifen-treated group. ${ }^{2}$

In the ABCSG-12 randomized trial, tamoxifen plus goserelin treatment was compared to anastrozole plus goserelin in the adjuvant setting in premenopausal breast cancer patients. The group receiving nonsteroidal AI plus goserelin had a 
higher frequency of arthralgia and bone pain, compared to the group receiving tamoxifen plus goserelin. In fact, the reported incidence of bone pain in the tamoxifen plus LHRH agonist group was $23 \%$, which is similar to our findings. ${ }^{9}$

As documented in large randomized prospective trials, AI in combination with LHRH agonists gave a higher incidence of arthralgia, compared to tamoxifen plus LHRH agonists. ${ }^{10}$ There may be several reasons behind this observation. AIs lower serum estrone, estradiol, and estrone sulfate levels. Deprivation of estrogen levels in the body is associated with decreased bone mineral density, osteoporosis, and increased incidence of arthritis. ${ }^{11}$ The same mechanism is also true in the case of loss of ovarian function by use of LHRH agonists that inhibit estrogen production in the ovaries. In the case of tamoxifen, its role as an estrogen agonist in the bone tissue of postmenopausal women is well established. ${ }^{12}$ It can be hypothesized that by this effect, tamoxifen may have an alleviating effect on bone and joint tissues in women with loss of ovarian function, and therefore, tamoxifen LHRH combination is less commonly associated with AHAMP.

An analysis was made for the identification of factors that are predictive for the development of AHAMP. In linear regression analysis, low BMI and smoking were associated with a higher risk of developing AHAMP. The relation between BMI and joint-related symptoms is complex. The incidence of arthralgia is reported to be higher in obese individuals (BMI >30) in the retrospective analysis of ATAC and IES trials. ${ }^{13,14}$

On the contrary, a trial assessing the incidence of arthralgia in patients receiving endocrine treatment with a design very similar to our own study reported the prevalence of arthralgia to be higher in individuals with a BMI $<25$, compared to individuals with BMI $25-30 .{ }^{4}$ In parallel to this observation, we also report that leaner individuals have a higher incidence of reported AHAMP. In addition, we also observed that lower BMI also correlated with increased pain severity. This finding could be explained by the fact that increased levels of sex hormones secreted by the adipose tissue in obese individuals could play a role in diminishing pain related to arthralgia.

Younger age was also found to be positively correlated with the intensity of the pain in a linear regression analysis model. The age factor is not commonly cited as a risk factor for developing AHAMP; however, some studies in the literature report the frequency of arthralgia symptoms to be higher in younger patients. ${ }^{15}$ It can be hypothesized that younger patients will be more sensitive to the adverse effects of the acute reduction of sex hormones due to hormonal treatment, when compared to their older counterparts. ${ }^{16}$ In this regard, younger patients are expected to experience AHAMP more intensely when compared to more elderly patients.

Estrogen receptors are found in joint cartilage, subchondral bone, and synovium. Estrogen is thought to have antiinflammatory and mild immunosuppressive properties. In vitro studies have documented that acute estrogen withdrawal leads to activation of nuclear factor $\kappa \mathrm{B}$ transcription factor, which, in turn, upregulates the production of proinflammatory cytokines ultimately leading to tissue destruction. ${ }^{17}$ Interestingly, estrogen receptors are also present in the limbic system and dorsal root ganglion. Estrogens play an antinociceptive role and inhibit pain signaling in the central nervous system. ${ }^{18}$ In the light of these data, it seems appropriate to suggest that the main reason for both younger age and lower BMI being linked to heightened intensity of joint symptoms is decreased sex hormone production in the body due to endocrine treatment.

Smoking is a well-established risk factor for bone fracture in women. It is reported to increase the cumulative risk of bone fracture by $50 \%$ in postmenopausal women. ${ }^{19}$ Cigarette smoking negatively influences estrogen production by inhibiting the activity of aromatase enzyme, increasing sex hormone binding globulins, and various other mechanisms. In addition, there is also evidence that by interfering with 25 hydroxylase enzyme activity and by promotion of chronic inflammation, it has deleterious effects on musculoskeletal system. ${ }^{20}$ However, the relation between cigarette smoking and AHAMP is not thoroughly assessed in the literature. In a retrospective analysis of the ATAC study, smoking was not found to be associated with carpal tunnel syndrome. ${ }^{21}$ In a different retrospective analysis of the same large study which assessed the risk factors for joint symptoms, cigarette smoking was found to be a risk factor in univariate analysis; however, it lost significance in multivariate analysis. ${ }^{13}$ In our study, AHAMP incidence was significantly higher in current smokers and smoking was positively correlated with more severe pain.

Whether low serum $25(\mathrm{OH}) \mathrm{D}(<20 \mathrm{ng} / \mathrm{mL})$ is a risk factor for developing AHAMP is a controversial issue. Interestingly, the prevalence of vitamin D deficiency in women with breast cancer undergoing chemotherapy can be as high as $70 \% .{ }^{22}$ In Turkey, even among healthy women, vitamin D deficiency is prevalent. In a recent study, $74 \%$ of the healthy women were found to be vitamin $\mathrm{D}$ deficient and mean serum $25(\mathrm{OH}) \mathrm{D}$ concentration was reported as $17 \mathrm{ng} / \mathrm{mL},{ }^{23}$ which is very close to the mean serum $25(\mathrm{OH}) \mathrm{D}$ concentration of our study group $(21.2 \mathrm{ng} / \mathrm{mL} \pm 7.1)$. 
In patients with osteomalacia (defined as serum $25(\mathrm{OH}) \mathrm{D}$ levels below $10 \mathrm{ng} / \mathrm{mL}$ ), bone pain and myalgia are wellestablished symptoms of the disease. In addition, serum vitamin $\mathrm{D}$ levels have a strong impact on bone mineral density, bone turnover markers, and parathyroid hormone levels. Therefore, low 25(OH)D concentrations are strongly linked with increased bone fracture rates and lower physical performance, especially in postmenopausal women. ${ }^{24,25}$

While some studies in the literature associated low $25(\mathrm{OH}) \mathrm{D}$ levels with only specific findings such as tendonitis developing in patients receiving antihormonal therapy, ${ }^{26}$ there are other studies that have not reported a correlation between low vitamin $\mathrm{D}$ concentrations and joint symptoms related to antihormonal treatment. ${ }^{27}$

We found that patients with joint-related pain symptoms had significantly lower 25(OH)D levels compared to their counterparts with no joint-related arthralgia. Lower vitamin D levels were also found to be associated with increased pain intensity. A study by Waltman et al is the first study to report an inverse correlation between muscle pain level and serum $25(\mathrm{OH}) \mathrm{D}$ concentration. ${ }^{28}$ Our study confirms the relation between AHAMP and serum 25(OH)D levels in patients with breast cancer receiving AI therapy.

Musculoskeletal adverse effects of adjuvant antihormonal agents that are used to treat breast cancer patients for prolonged time periods can have a negative impact on their quality of daily life. There are only very few studies in the literature assessing the impact of musculoskeletal pain related to antihormonal treatment on the HRQOL in breast cancer survivors. A study by Olufade et al, which claims to be the first study assessing HRQOL in patients treated with AIs in the adjuvant setting, has reported worse physical component scores in patients with AHAMP, while mental component scores were not found to be significantly affected. ${ }^{29}$

In parallel to this study, we also report that HRQOL was found to be significantly worse (measured by utilizing both SF-36 and FACT-B questionnaires) in patients with AHAMP. While only physical component scores were statistically lower in SF-36, both mental and physical scores were significantly worse in patients with joint symptoms when analysis was made with FACT-B questionnaire (Table 4). In our view, FACT-B, which includes the compartments specifically related to worry and symptoms about breast cancer, enables a more specific assessment for studying the effects of treatment-related adverse effects on HRQOL in breast cancer survivors.

All the patients in the study group received taxane-containing chemotherapy regimens before starting antihormonal treatment. Prior taxane exposure is associated with an increased risk of developing musculoskeletal symptoms during antihormonal therapies. ${ }^{4}$

Whether the severity of joint symptoms and the effect of AHAMP on HRQOL are lower in breast cancer patients who have not received prior chemotherapy remain to be elucidated. The relatively small sample size of the study and the lack of a control arm of patients who have not received antihormonal treatment after surgery for breast cancer should be taken into consideration when interpreting the results of the study.

A homogeneous patient group with regard to disease stage and prior therapies received before the start of antihormonal treatment, inclusion of a rheumatology specialist for evaluation of musculoskeletal symptoms, and utilization of a validated questionnaire for assessment of HRQOL in breast cancer patients are the strong points of the study.

\section{Conclusion}

Antihormonal treatment associated musculoskeletal symptoms seem to related to estrogen withdrawal as a result of aromatase inhibition or functional ovarian suppression. Certain risk factors such as low BMI, cigarette smoking, and low serum 25(OH)D levels have been identified in this study. Treating physicians should be aware of this common and discomforting treatment-related side effect and be open to discuss this issue with the patients. Recognition of patients who are at risk of developing joint-related complaints and development of therapies aimed at alleviation of pain and discomfort should be the main targets for future research.

\section{Disclosure}

The authors report no conflicts of interest in this work.

\section{References}

1. Coates AS, Keshaviah A, Thurlimann B, et al. Five years of letrozole compared with tamoxifen as initial adjuvant therapy for postmenopausal women with endocrine-responsive early breast cancer: update of study BIG 1-98. J Clin Oncol. 2007;25(5):486-492.

2. Bernhard J, Luo W, Ribi K, et al. Patient-reported outcomes with adjuvant exemestane versus tamoxifen in premenopausal women with early breast cancer undergoing ovarian suppression (TEXT and SOFT): a combined analysis of two phase 3 randomised trials. Lancet Oncol. 2015;16(7): 848-858.

3. Nyrop KA, Callahan LF, Rini C, et al. Adaptation of an evidence-based arthritis program for breast cancer survivors on aromatase inhibitor therapy who experience joint pain. Prev Chronic Dis. 2015;12:E91.

4. Crew KD, Greenlee H, Capodice J, et al. Prevalence of joint symptoms in postmenopausal women taking aromatase inhibitors for early-stage breast cancer. J Clin Oncol. 2007;25(25):3877-3883.

5. Hershman DL, Shao T, Kushi LH, et al. Early discontinuation and nonadherence to adjuvant hormonal therapy are associated with increased mortality in women with breast cancer. Breast Cancer Res Treat. 2011; 126(2):529-537. 
6. Niravath P. Aromatase inhibitor-induced arthralgia: a review. Ann Oncol. 2013;24(6):1443-1449.

7. Pinar R. Reliability and construct validity of the SF-36 in Turkish cancer patients. Qual Life Res. 2005;14(1):259-264.

8. Bonomi AE, Cella DF, Hahn EA, et al. Multilingual translation of the Functional Assessment of Cancer Therapy (FACT) quality of life measurement system. Qual Life Res. 1996;5(3):309-320.

9. Gnant M, Mlineritsch B, Stoeger H, et al. Adjuvant endocrine therapy plus zoledronic acid in premenopausal women with early-stage breast cancer: 62-month follow-up from the ABCSG-12 randomised trial. Lancet Oncol. 2011;12(7):631-641.

10. Bernhard J, Luo W, Ribi K, et al. Patient-reported endocrine symptoms, sexual functioning, and quality of life (QoL) in the IBCSG TEXT and SOFT trials: Adjuvant treatment with exemestane (E) plus ovarian function suppression (OFS) versus tamoxifen (T) plus OFS in premenopausal women with hormone receptor-positive $(\mathrm{HR}+)$ early breast cancer (BC). 2014 ASCO Annual Meeting Citation. J Clin Oncol. 2014; 32:5s(suppl; abstr 557).

11. Felson DT, Cummings SR. Aromatase inhibitors and the syndrome of arthralgias with estrogen deprivation. Arthritis Rheum. 2005;52(9): 2594-2598.

12. Resch A, Biber E, Seifert M, Resch H. Evidence that tamoxifen preserves bone density in late postmenopausal women with breast cancer. Acta Oncol. 1998;37(7-8):661-664.

13. Sestak I, Cuzick J, Sapunar F, et al. Risk factors for joint symptoms in patients enrolled in the ATAC trial: a retrospective, exploratory analysis. Lancet Oncol. 2008;9(9):866-872.

14. Mieog JS, Morden JP, Bliss JM, Coombes RC, van de Velde CJ. Carpal tunnel syndrome and musculoskeletal symptoms in postmenopausal women with early breast cancer treated with exemestane or tamoxifen after 2-3 years of tamoxifen: a retrospective analysis of the Intergroup Exemestane Study. Lancet Oncol. 2012;13(4):420-432.

15. Cella D, Fallowfield LJ. Recognition and management of treatmentrelated side effects for breast cancer patients receiving adjuvant endocrine therapy. Breast Cancer Res Treat. 2008;107(2):167-180.

16. Menas P, Merkel D, Hui W, Lawton J, Harper A, Carro G. Incidence and management of arthralgias in breast cancer patients treated with aromatase inhibitors in an outpatient oncology clinic. J Oncol Pharm Pract. 2012;18(4):387-393.

17. Weitzmann MN. The role of inflammatory cytokines, the RANKL/ OPG axis, and the immunoskeletal interface in physiological bone turnover and osteoporosis. Scientifica (Cairo). 2013;2013:125705.
18. Martin-Millan M, Castaneda S. Estrogens, osteoarthritis and inflammation. Joint Bone Spine. 2013;80(4):368-373.

19. Law MR, Hackshaw AK. A meta-analysis of cigarette smoking, bone mineral density and risk of hip fracture: recognition of a major effect. BMJ. 1997;315(7112):841-846.

20. Abate M, Vanni D, Pantalone A, Salini V. Cigarette smoking and musculoskeletal disorders. Muscles Ligaments Tendons J. 2013;3(2):63-69.

21. Sestak I, Sapunar F, Cuzick J. Aromatase inhibitor-induced carpal tunnel syndrome: results from the ATAC trial. J Clin Oncol. 2009; 27(30):4961-4965.

22. Crew KD, Shane E, Cremers S, McMahon DJ, Irani D, Hershman DL. High prevalence of vitamin D deficiency despite supplementation in premenopausal women with breast cancer undergoing adjuvant chemotherapy. J Clin Oncol. 2009;27(13):2151-2156.

23. Hekimsoy Z, Dinc G, Kafesciler S, et al. Vitamin D status among adults in the Aegean region of Turkey. BMC Public Health. 2010;10:782.

24. Kuchuk NO, Pluijm SM, van Schoor NM, Looman CW, Smit JH, Lips P. Relationships of serum 25-hydroxyvitamin D to bone mineral density and serum parathyroid hormone and markers of bone turnover in older persons. J Clin Endocrinol Metab. 2009;94(4):1244-1250.

25. Martin-Herranz A, Salinas-Hernandez P. Vitamin D supplementation review and recommendations for women diagnosed with breast or ovary cancer in the context of bone health and cancer prognosis/risk. Crit Rev Oncol Hematol. 2015;96(1):91-99.

26. Singer O, Cigler T, Moore AB, Levine AB, Do HT, Mandl LA. Hypovitaminosis $\mathrm{D}$ is a predictor of aromatase inhibitor musculoskeletal symptoms. Breast J. 2014;20(2):174-179.

27. Laroche F, Coste J, Medkour T, et al. Classification of and risk factors for estrogen deprivation pain syndromes related to aromatase inhibitor treatments in women with breast cancer: a prospective multicenter cohort study. J Pain. 2014;15(3):293-303.

28. Waltman NL, Ott CD, Twiss JJ, Gross GJ, Lindsey AM. Vitamin D insufficiency and musculoskeletal symptoms in breast cancer survivors on aromatase inhibitor therapy. Cancer Nurs. 2009;32(2):143-150.

29. Olufade T, Gallicchio L, MacDonald R, Helzlsouer KJ. Musculoskeletal pain and health-related quality of life among breast cancer patients treated with aromatase inhibitors. Support Care Cancer. 2015; 23(2):447-455.
OncoTargets and Therapy

\section{Publish your work in this journal}

OncoTargets and Therapy is an international, peer-reviewed, open access journal focusing on the pathological basis of all cancers, potential targets for therapy and treatment protocols employed to improve the management of cancer patients. The journal also focuses on the impact of management programs and new therapeutic agents and protocols on

\section{Dovepress}

patient perspectives such as quality of life, adherence and satisfaction. The manuscript management system is completely online and includes a very quick and fair peer-review system, which is all easy to use. Visit http://www.dovepress.com/testimonials.php to read real quotes from published authors. 